\title{
Sum rule for the twist four longitudinal structure function
}

\author{
A. Harindranath ${ }^{a, b}$, Rajen Kundu ${ }^{a}$, Asmita Mukherjee ${ }^{a}$, and James P. Vary ${ }^{b, c}$ \\ ${ }^{a}$ Saha Institute of Nuclear Physics, 1/AF Bidhan Nagar, Calcutta, 700064 India \\ ${ }^{b}$ International Institute of Theoretical and Applied Physics, Iowa State University, \\ 123 Office and Lab Link, Ames, IA 50011, U.S.A. \\ ${ }^{c}$ Department of Physics and Astronomy, Iowa State University, Ames, IA 50011, U.S.A.
}

(October 2, 1997)

\begin{abstract}
We investigate the twist four longitudinal structure function $F_{L}^{\tau=4}$ of deep inelastic scattering and show that the integral of $\frac{F_{L}^{\tau=4}}{x}$ is related to the expectation value of the fermionic part of the light-front Hamiltonian density at fixed momentum transfer. We show that the new relation, in addition to providing physical intuition on $F_{L}^{\tau=4}$, relates the quadratic divergences of $F_{L}^{\tau=4}$ to the quark mass correction in light-front QCD and hence provides a new pathway for the renormalization of the corresponding twist four operator. The mixing of quark and gluon operators in QCD naturally leads to a twist four longitudinal gluon structure function and to a new sum rule $\int d x \frac{F_{L}}{x}=4 \frac{M^{2}}{Q^{2}}$, which is the first sum rule obtained for a twist four observable. The validity of the sum rule in a non-perturbative context is explicitly verified in two-dimensional QCD.
\end{abstract}

PACS: 11.10.Ef, 11.10.Gh, 11.55.Hx, 13.40.-f

Keywords: twist four longitudinal structure function, light-front QCD Hamiltonian, sum rule, quadratic divergences, renormalization

\section{Introduction}

Measurements [四] of $R=\frac{\sigma_{L}}{\sigma_{T}}$ show [2] that dynamical higher twist effects play an important role in nucleon structure experiments in the SLAC kinematic range. Indeed, twist four ("power suppressed") contributions to the deep inelastic structure functions contain non-trivial, non-perturbative information on the structure of hadrons [3]. So far, however, a clear and simple physical picture of twist four contributions is lacking. In addition, power counting indicates that in the bare theory, twist four-matrix elements will be afflicted with

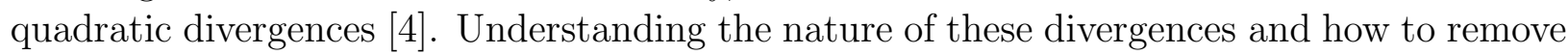
them (renormalization) is essential in order to compare with the experimental data.

At twist two level, light-front analysis of deep inelastic scattering provides an intuitive physical picture of various structure functions. Recently, the extraction of the unpolarized twist two structure function $F_{2}$ from the transverse component of the bilocal matrix element [5] and the physical picture of the transverse polarized structure function $g_{T}$ and associated 
issues [6,7] have been analyzed in a light-front field theory approach [8]. The method uses Fock space expansion for the Hamiltonian which is physically intuitive and is straightforward to calculate.

To resolve the outstanding issues mentioned above, we use this same framework to evaluate the twist four contribution to the longitudinal structure function. The resulting relationship with the fermionic part of the light-front Hamiltonian density provides a convenient pathway to renormalization and leads to a definition of a twist four longitudinal gluon structure function. We then find a sum rule, the first one at the twist four level.

The BJL limit, together with light-front current algebra [9] in $A^{+}=0$ gauge, the tools used in the pre-QCD era, lead to the twist four part of the fermionic contribution to the longitudinal structure function

$$
F_{L(f)}^{\tau=4}(x)=i \frac{1}{Q^{2}} \frac{\left(x P^{+}\right)^{2}}{\pi} \int d y^{-} e^{-\frac{i}{2} P^{+} y^{-} x}\left\langle P\left|\overline{\mathcal{J}}^{-}(y \mid 0)\right| P\right\rangle-4 \frac{\left(P^{\perp}\right)^{2}}{Q^{2}} x F_{2(f)}(x)
$$

where $f$ represents a quark $(q)$ or anti-quark $(\bar{q})$ or both depending on the target $|P\rangle$, and where the twist two contribution to the $F_{2(f)}$ structure function

$$
\frac{F_{2(f)}(x)}{x}=i \frac{1}{4 \pi} \int d y^{-} e^{-\frac{i}{2} P^{+} y^{-} x}\left\langle P\left|\bar{J}^{+}(y \mid 0)\right| P\right\rangle .
$$

The bilocal current operator,

$$
\overline{\mathcal{J}}^{\mu}(y \mid 0)=\frac{1}{2 i}\left[\bar{\psi}(y) \gamma^{\mu} \psi(0)-\bar{\psi}(0) \gamma^{\mu} \psi(y)\right] .
$$

Note that in the case of quark (anti-quark) contributions, the second (first) term in the expression for the bilocal current in Eq. (3) vanishes. We have,

$$
F_{L(q)}^{\tau=4}(x)=\mathcal{M}_{1}+\mathcal{M}_{2}
$$

with

$$
\mathcal{M}_{1}=\frac{1}{Q^{2}} \frac{x^{2}\left(P^{+}\right)^{2}}{2 \pi} \int d y^{-} e^{-\frac{i}{2} P^{+} y^{-} x}\left\langle P\left|\bar{\psi}\left(y^{-}\right) \gamma^{-} \psi(0)\right| P\right\rangle
$$

and

$$
\mathcal{M}_{2}=-\frac{\left(P^{\perp}\right)^{2}}{\left(P^{+}\right)^{2}} \frac{1}{Q^{2}} \frac{x^{2}\left(P^{+}\right)^{2}}{2 \pi} \int d y^{-} e^{-\frac{i}{2} P^{+} y^{-} x}\left\langle P\left|\bar{\psi}\left(y^{-}\right) \gamma^{+} \psi(0)\right| P\right\rangle .
$$

Using $\bar{\psi}\left(y^{-}\right) \gamma^{-} \psi(0)=2 \psi^{-\dagger}\left(y^{-}\right) \psi^{-}(0)$, where

$$
\psi^{-}(z)=\frac{1}{4 i} \int d y^{-} \epsilon\left(z^{-}-y^{-}\right)\left[\alpha^{\perp} \cdot\left(i \partial^{\perp}+g A^{\perp}\right)+\gamma^{0} m\right] \psi^{+}\left(y^{-}\right),
$$

and $\epsilon\left(x^{-}\right)=-\frac{i}{\pi} P \int \frac{d \omega}{\omega} e^{\frac{i}{2} \omega x^{-}}$, we arrive at

$$
\begin{aligned}
\mathcal{M}_{1}= & \frac{1}{\pi Q^{2}} \int d y^{-} e^{-\frac{i}{2} P^{+} y^{-} x}\langle P| \psi^{+\dagger}\left(y^{-}\right) \\
& {\left[\alpha^{\perp} \cdot\left[i \partial^{\perp}+g A^{\perp}(y)\right]+\gamma^{0} m\right]\left[\alpha^{\perp} \cdot\left[i \partial^{\perp}+g A^{\perp}(0)\right]+\gamma^{0} m\right] \psi^{+}(0)|P\rangle, }
\end{aligned}
$$


and

$$
\mathcal{M}_{2}=-2 \frac{\left(P^{\perp}\right)^{2}}{Q^{2}} \frac{1}{2 \pi} x^{2} \int d y^{-} e^{-\frac{i}{2} P^{+} y^{-} x}\left\langle P\left|\psi^{+\dagger}\left(y^{-}\right) \psi^{+}(0)\right| P\right\rangle
$$

Thus we have obtained an expression for the twist four part of the fermionic component of the longitudinal structure function. After the establishment of QCD as the underlying theory

of strong interactions, the twist four part of the quark contributions to the longitudinal structure function has been given in the limits of vanishing target transverse momentum and massless quark and in the $A^{+}=0$ gauge using the QCD factorization method [10,11]

$$
\begin{aligned}
& F_{L(q)}^{\tau=4}(x)=\frac{1}{\pi Q^{2}} \int d y^{-} e^{-\frac{i}{2} P^{+} y^{-} x}\langle P| \psi^{+^{\dagger}}\left(y^{-}\right) \\
& {\left[i \partial^{\perp}+g A^{\perp}(y)\right] \cdot \alpha^{\perp} \alpha^{\perp} \cdot\left[i \partial^{\perp}+g A^{\perp}(0)\right] \psi^{+}(0)|P\rangle .}
\end{aligned}
$$

Using Eqs. (4), (8), and (9), and taking the limit of vanishing target transverse momentum and massless quark, our result given in Eq. (1) reduces to that obtained via the QCD factorization method (Eq. 10).

Note that the expression for $F_{L(q)}^{\tau=4}$ given in Eq. (11) appears to violate transverse boost invariance. But, we exhibit below with explicit calculations in Sec. 3 that the $P^{\perp}$ dependence cancels between Eqs. (8) and (9) so that the full $F_{L(q)}^{\tau=4}$ is indeed boost invariant.

\section{Sum rule for the twist four longitudinal structure function}

From Eq. (2) it follows that $F_{2(f)}(-x)=F_{2(f)}(x)$ and from Eq. (11) we explicitly find that $F_{L(f)}^{\tau=4}(-x)=-F_{L(f)}^{\tau=4}(x)$. Consider the integral

$$
\begin{aligned}
\int_{-\infty}^{+\infty} d x \frac{F_{L(f)}^{\tau=4}(x)}{x}= & 2 \int_{0}^{\infty} d x \frac{F_{L(f)}^{\tau=4}(x)}{x} \\
= & \int_{-\infty}^{+\infty} \frac{d x}{x}\left[i \frac{1}{Q^{2}} \frac{\left(x P^{+}\right)^{2}}{\pi} \int d y^{-} e^{-\frac{i}{2} P^{+} y^{-} x}\right. \\
& \left.\times\left[\left\langle P\left|\overline{\mathcal{J}}^{-}(y \mid 0)\right| P\right\rangle-\frac{\left(P^{\perp}\right)^{2}}{\left(P^{+}\right)^{2}}\left\langle P\left|\overline{\mathcal{J}}^{+}(y \mid 0)\right| P\right\rangle\right]\right]
\end{aligned}
$$

Interchanging the orders of $x$ and $y^{-}$integrations and carrying out the integrations explicitly, we arrive at $[12$

$$
\int_{-\infty}^{+\infty} d x \frac{F_{L(f)}^{\tau=4}\left(x, Q^{2}\right)}{x}=\frac{4}{Q^{2}}\left[\left\langle P\left|i \bar{\psi} \gamma^{-} \partial^{+} \psi\right|_{(0)} \mid P\right\rangle-\frac{\left(P^{\perp}\right)^{2}}{\left(P^{+}\right)^{2}}\left\langle P\left|i \bar{\psi} \gamma^{+} \partial^{+} \psi\right|_{(0)} \mid P\right\rangle\right] .
$$

Identifying $i \bar{\psi} \gamma^{-} \partial^{+} \psi=\theta_{q}^{+-}$, the fermionic part of the light-front QCD Hamiltonian density and $i \bar{\psi} \gamma^{+} \partial^{+} \psi=\theta_{q}^{++}$, the fermionic part of the light-front QCD longitudinal momentum density, (see Eqs. (15) and (17) below), we have:

$$
\int_{0}^{1} d x \frac{F_{L(f)}^{\tau=4}\left(x, Q^{2}\right)}{x}=\frac{2}{Q^{2}}\left[\left\langle P\left|\theta_{q}^{+-}(0)\right| P\right\rangle-\frac{\left(P^{\perp}\right)^{2}}{\left(P^{+}\right)^{2}}\left\langle P\left|\theta_{q}^{++}(0)\right| P\right\rangle\right],
$$


where we have used the fact that the physical structure function vanishes for $x>1$.

The integral of $\frac{F_{L(f)}^{\tau=4}}{x}$ is therefore related to the hadron matrix element of the (gauge invariant) fermionic part of the light-front Hamiltonian density. This result manifests the physical content and the non-perturbative nature of the twist-four part of the longitudinal structure function.

The fermionic operator matrix elements appearing in Eq. (13) change with $Q^{2}$ as a result of the mixing of quark and gluon operators in QCD under renormalization. Next we analyze the operator mixing and derive a new sum rule at the twist four level.

The symmetric, gauge-invariant energy-momentum tensor in QCD is given by

$$
\begin{aligned}
\theta^{\mu \nu}= & \frac{1}{2} \bar{\psi} i\left[\gamma^{\mu} D^{\nu}+\gamma^{\nu} D^{\mu}\right] \psi-F^{\mu \lambda a} F_{\lambda a}^{\nu}+\frac{1}{4} g^{\mu \nu}\left(F_{\lambda \sigma a}\right)^{2} \\
& -g^{\mu \nu} \bar{\psi}\left(i \gamma^{\lambda} D_{\lambda}-m\right) \psi .
\end{aligned}
$$

The last term vanishes using the equation of motion.

Formally, we split the energy momentum tensor into a "fermionic" part $\theta_{q}^{\mu \nu}$ representing the first term in Eq. (14) and a "gauge bosonic" part $\theta_{g}^{\mu \nu}$ representing the second and third terms in Eq. (14). To be consistent with the study of deep inelastic structure function formulated in the $A^{+}=0$ gauge, we shall work in the same gauge. We have, for the fermionic part of the longitudinal momentum density,

$$
\theta_{q}^{++}=i \bar{\psi} \gamma^{+} \partial^{+} \psi
$$

For the fermionic part of the Hamiltonian density, we have,

$$
\theta_{q}^{+-}=i \psi^{+\dagger} \partial^{-} \psi^{+}+g \psi^{+\dagger} A^{-} \psi^{+}+i \psi^{-\dagger} \partial^{+} \psi^{-}
$$

Using the Dirac equation for the fermion, we find that the sum of the first two terms equals the third term in the above equation. Therefore,

$$
\begin{aligned}
\theta_{q}^{+-} & =i \bar{\psi} \gamma^{-} \partial^{+} \psi=2 i \psi^{-\dagger} \partial^{+} \psi^{-} \\
& =2 \psi^{+\dagger}\left[\alpha^{\perp} \cdot\left(i \partial^{\perp}+g A^{\perp}\right)+\gamma^{0} m\right] \frac{1}{i \partial^{+}}\left[\alpha^{\perp} \cdot\left(i \partial^{\perp}+g A^{\perp}\right)+\gamma^{0} m\right] \psi^{+} .
\end{aligned}
$$

The gauge boson part of the Hamiltonian density is given by

$$
\begin{aligned}
\theta_{g}^{+-}=-F^{+\lambda a} F_{\lambda a}^{-}+\frac{1}{4} g^{+-}\left(F_{\lambda \sigma a}\right)^{2}=\frac{1}{4}\left(\partial^{+} A^{-a}\right)^{2}+\frac{1}{2} F^{i j a} F_{i j}^{a} \\
=\left(\partial^{i} A_{a}^{j}\right)^{2}+2 g f^{a b c} A_{a}^{i} A_{b}^{j} \partial^{i} A_{c}^{j}+\frac{g^{2}}{2} f^{a b c} f^{a d e} A_{b}^{i} A_{c}^{j} A_{d}^{i} A_{e}^{j} \\
+2 g \partial^{i} A_{a}^{i}\left(\frac{1}{\partial^{+}}\right)\left(f^{a b c} A_{b}^{j} \partial^{+} A_{c}^{j}+2\left(\psi^{+}\right)^{\dagger} T^{a} \psi^{+}\right) \\
+g^{2}\left(\frac{1}{\partial^{+}}\right)\left(f^{a b c} A_{b}^{i} \partial^{+} A_{c}^{i}+2\left(\psi^{+}\right)^{\dagger} T^{a} \psi^{+}\right)\left(\frac{1}{\partial^{+}}\right)\left(f^{a d e} A_{d}^{j} \partial^{+} A_{e}^{j}+2\left(\psi^{+}\right)^{\dagger} T^{a} \psi^{+}\right)
\end{aligned}
$$

where we have used the equation of constraint for the gauge field.

We define the twist four longitudinal gluon structure function 


$$
\begin{aligned}
F_{L(g)}^{\tau=4}(x)= & \frac{1}{Q^{2}} \frac{x P^{+}}{2 \pi} \int d y^{-} e^{-\frac{i}{2} P^{+} y^{-} x} \\
& \quad\left[\left\langle P\left|(-) F^{+\lambda a}\left(y^{-}\right) F_{\lambda a}^{-}(0)+\frac{1}{4} g^{+-} F^{\lambda \sigma a}\left(y^{-}\right) F_{\lambda \sigma a}(0)\right| P\right\rangle\right. \\
& \left.\quad-\frac{\left(P^{\perp}\right)^{2}}{\left(P^{+}\right)^{2}}\left\langle P\left|F^{+\lambda a}\left(y^{-}\right) F_{\lambda a}^{+}(0)\right| P\right\rangle\right] .
\end{aligned}
$$

Then we have,

$$
\int_{0}^{1} \frac{d x}{x}\left[F_{L(q)}^{\tau=4}+F_{L(g)}^{\tau=4}\right]=\int_{0}^{1} \frac{d x}{x} F_{L}^{\tau=4}=\frac{2}{Q^{2}}\left[\left\langle P\left|\theta^{+-}(0)\right| P\right\rangle-\frac{\left(P^{\perp}\right)^{2}}{\left(P^{+}\right)^{2}}\left\langle P\left|\theta^{++}(0)\right| P\right\rangle\right] .
$$

But,

$$
\left\langle P\left|\theta^{+-}(0)\right| P\right\rangle=2 P^{+} P^{-}=2\left(M^{2}+\left(P^{\perp}\right)^{2}\right) \text { and }\left\langle P\left|\theta^{++}(0)\right| P\right\rangle=2\left(P^{+}\right)^{2},
$$

where $M$ is the invariant mass of the hadron. Thus we arrive at the new sum rule for the twist four part of the longitudinal structure function

$$
\int_{0}^{1} \frac{d x}{x} F_{L}^{\tau=4}=4 \frac{M^{2}}{Q^{2}}
$$

To our knowledge, this is the first sum rule at the twist four level of deep inelastic scattering or for QCD in general. 


\section{Implications of the sum rule}

Next, we investigate the implications of Eq. (13) for quadratic divergences in $F_{L(q)}^{\tau=4}$. For simplicity, we select a dressed quark target and evaluate the structure functions to order $g^{2}$. That is, we take the state $|P\rangle$ to be a dressed quark consisting of bare states of a quark and a quark plus a gluon:

$$
\begin{aligned}
|P, \sigma\rangle= & \phi_{1} b^{\dagger}(P, \sigma)|0\rangle \\
+ & \sum_{\sigma_{1}, \lambda_{2}} \int \frac{d k_{1}^{+} d^{2} k_{1}^{\perp}}{\sqrt{2(2 \pi)^{3} k_{1}^{+}}} \int \frac{d k_{2}^{+} d^{2} k_{2}^{\perp}}{\sqrt{2(2 \pi)^{3} k_{2}^{+}}} \sqrt{2(2 \pi)^{3} P^{+}} \delta^{3}\left(P-k_{1}-k_{2}\right) \\
& \phi_{2}\left(P, \sigma \mid k_{1}, \sigma_{1} ; k_{2}, \lambda_{2}\right) b^{\dagger}\left(k_{1}, \sigma_{1}\right) a^{\dagger}\left(k_{2}, \lambda_{2}\right)|0\rangle .
\end{aligned}
$$

Without loss of generality, we work in the massless limits for the dressed and bare quarks to obtain

$$
\begin{aligned}
\mathcal{M}_{1}= & \frac{1}{\pi Q^{2}} \int d y^{-} e^{-\frac{i}{2} P^{+} y^{-} x}\langle P| \psi^{+^{\dagger}}\left(y^{-}\right) \\
& {\left[i \partial^{\perp}+g A^{\perp}(y)\right] \cdot \alpha^{\perp} \alpha^{\perp} \cdot\left[i \partial^{\perp}+g A^{\perp}(0)\right] \psi^{+}(0)|P\rangle, }
\end{aligned}
$$

and

$$
\mathcal{M}_{2}=-4 \frac{\left(P^{\perp}\right)^{2}}{Q^{2}} x F_{2(q)}(x) .
$$

Note that the matrix elements appearing in Eqs. (8), (9), (18), and (19) involve products of operators. In this initial study of quadratic divergences we treat them as normal ordered which is sufficient for our purposes here. This aspect of the problem merits further investigation which we hope to undertake in the near future. However, the terms we drop are quadratically divergent and they will affect only the counterterm structure.

First we evaluate the contribution $\mathcal{M}_{2}$ given in Eq. (26). We obtain,

$$
\mathcal{M}_{2}=-4 C_{f} \frac{\left(P^{\perp}\right)^{2}}{Q^{2}} x^{2}\left[\delta(1-x)+\frac{\alpha_{s}}{2 \pi} \ln \Lambda^{2}\left[\frac{1+x^{2}}{1-x}-\delta(1-x) \int d y \frac{1+y^{2}}{1-y}\right]\right]
$$

where we have cutoff the transverse momentum integral at $\Lambda$. Note that the result in Eq. (27) violates transverse boost invariance.

We have, from Eq. (25),

$$
\begin{aligned}
\mathcal{M}_{1}= & -\frac{1}{\pi Q^{2}} \int d y^{-} e^{-\frac{i}{2} P^{+} y^{-} x}\left\langle P\left|\psi^{+\dagger}\left(y^{-}\right)\left(\partial^{\perp}\right)^{2} \psi^{+}(0)\right| P\right\rangle \\
& +g \frac{1}{\pi Q^{2}} \int d y^{-} e^{-\frac{i}{2} P^{+} y^{-} x}\left\langle P\left|\psi^{+\dagger}\left(y^{-}\right) i \partial^{\perp} \cdot \alpha^{\perp} \alpha^{\perp} \cdot A^{\perp}(0) \psi^{+}(0)\right| P\right\rangle \\
& +g \frac{1}{\pi Q^{2}} \int d y^{-} e^{-\frac{i}{2} P^{+} y^{-} x}\left\langle P\left|\psi^{+\dagger}\left(y^{-}\right) \alpha^{\perp} \cdot A^{\perp}(y) i \partial^{\perp} \cdot \alpha^{\perp} \psi^{+}(0)\right| P\right\rangle \\
& +g^{2} \frac{1}{\pi Q^{2}} \int d y^{-} e^{-\frac{i}{2} P^{+} y^{-} x}\left\langle P\left|\psi^{+\dagger}\left(y^{-}\right) A^{\perp}(y) \cdot A^{\perp}(0)\right| P\right\rangle \\
\equiv & \mathcal{M}_{1}^{a}+\mathcal{M}_{1}^{b}+\mathcal{M}_{1}^{c}+\mathcal{M}_{1}^{d} .
\end{aligned}
$$


Since the operators in Eq. (25) are taken to be normal ordered, the contribution of $\mathcal{M}_{1}^{d}$ vanishes to order $g^{2}$.

Explicit calculation leads to the diagonal Fock basis contributions

$$
\begin{aligned}
\left(\mathcal{M}_{1}\right)_{\text {diag }}=\mathcal{M}_{1}^{a}=4 C_{f} & \frac{\left(P^{\perp}\right)^{2}}{Q^{2}} x^{2}\left[\delta(1-x)+\frac{\alpha_{s}}{2 \pi} \ln \Lambda^{2}\left[\frac{1+x^{2}}{1-x}-\delta(1-x) \int d y \frac{1+y^{2}}{1-y}\right]\right] \\
+ & C_{f} \frac{g^{2}}{2 \pi^{2}} \frac{\Lambda^{2}}{Q^{2}} \frac{1+x^{2}}{1-x} .
\end{aligned}
$$

The first term here explicitly cancels the term $\mathcal{M}_{2}$ given in Eq. (27).

Off-diagonal contributions

$$
\left(\mathcal{M}_{1}\right)_{\text {nondiag }}=\mathcal{M}_{1}^{b}+\mathcal{M}_{1}^{c}=-C_{f} \frac{g^{2}}{\pi^{2}} \frac{\Lambda^{2}}{Q^{2}} \frac{1}{1-x} .
$$

Adding all the contributions, we have,

$$
F_{L(q)}^{\tau=4}(x)=-C_{f} \frac{g^{2}}{2 \pi^{2}} \frac{\Lambda^{2}}{Q^{2}}(1+x)
$$

As anticipated from power counting, we have generated quadratic divergences for $F_{L}$ in the bare theory. Now we are faced with two issues: (a) What is the principle for adding counterterms? (b) What determines the finite parts of the counterterms?

Further, since $F_{L(q)}^{\tau=4}$ is directly related to the physical longitudinal cross section, we expect $F_{L(q)}^{\tau=4}$ to be positive definite (see, for example, Ref. [10]). From our answers we see that we get a negative answer which is free from end point singularities but is quadratically divergent. Note that it is easy to show from our expressions in Eqs. (8) and (9) for a free quark of mass $m, F_{L(q)}^{\tau=4}=4 \frac{m^{2}}{Q^{2}} \delta(1-x)$, a well-known result.

Now we show that the sum rule relating the integral of $\frac{F_{L(q)}^{\tau=4}(x)}{x}$ to the fermionic part of the light-front Hamiltonian density helps us to understand the results we obtained for $F_{L}$ for a dressed quark in the bare theory and indicates how to add counterterms to renormalize the theory.

A straightforward evaluation leads to

$$
\begin{gathered}
\left\langle P\left|\theta_{q}^{+-}(0)\right| P\right\rangle_{\text {nondiag }}=-C_{f} \frac{1}{2} \frac{g^{2}}{\pi^{2}} \Lambda^{2} \int_{0}^{1} \frac{d x}{x} \frac{1}{1-x}, \\
\left\langle P\left|\theta_{q}^{+-}(0)\right| P\right\rangle_{\text {diag }}-\frac{\left(P^{\perp}\right)^{2}}{\left(P^{+}\right)^{2}}\left\langle P\left|\theta_{q}^{++}(0)\right| P\right\rangle_{\text {diag }}=C_{f} \frac{1}{2} \frac{g^{2}}{2 \pi^{2}} \Lambda^{2} \int_{0}^{1} \frac{d x}{x} \frac{1+x^{2}}{1-x},
\end{gathered}
$$

Adding the diagonal and off-diagonal contributions from the fermionic part of the Hamiltonian density, we arrive at

$$
\frac{2}{Q^{2}}\left[\left\langle P\left|\theta_{q}^{+-}(0)\right| P\right\rangle-\frac{\left(P^{\perp}\right)^{2}}{\left(P^{+}\right)^{2}}\left\langle P\left|\theta_{q}^{++}(0)\right| P\right\rangle\right]=-C_{f} \frac{g^{2}}{2 \pi^{2}} \frac{\Lambda^{2}}{Q^{2}} \int_{0}^{1} \frac{d x}{x}(1+x) .
$$

Comparison of Eqs. (32) and (35) immediately shows that the relation given in Eq. (13) has been successfully tested to order $g^{2}$ in perturbative QCD in the bare theory. 
Apart from the verification of the sum rule, the dressed quark calculation serves to illustrate the physical origin of quadratic divergences in the corresponding twist four matrix element. The sum rule explicitly relates the quadratic divergences in $F_{L(q)}^{\tau=4}$ to the fermion mass shift in light-front Hamiltonian perturbation theory. The quadratic divergence is to be removed by adding counterterms. The precise selection of counterterms is dictated by the regularization and renormalization of the light-front QCD Hamiltonian, the diagonalization of which results in the hadronic structure. The choice of counterterms in the Hamiltonian, in turn, dictates the counterterms to be added to the longitudinal structure function which results in the prediction of theoretical twist four contribution to $F_{L}$.

To complete the discussion of the fermion mass shift, we now consider the contributions from the gluonic part of the Hamiltonian density.

Take the off-diagonal contribution arising from

$$
\mathcal{M}_{3}=-\left\langle P\left|4 g \frac{1}{\partial^{+}}\left(\partial^{i} A^{i a}\right) \psi^{+\dagger} T^{a} \psi^{+}\right| P\right\rangle .
$$

A straightforward evaluation leads to

$$
\mathcal{M}_{3}=-C_{f} \frac{g^{2}}{2 \pi^{2}} \Lambda^{2} \int_{0}^{1} \frac{d x}{x}\left[\frac{2 x^{2}}{(1-x)^{2}}+\frac{x}{1-x}\right]
$$

Thus from Eqs. (33) and (37) we have,

$$
\left\langle P\left|\left[\theta_{q}^{+-}(0)+\theta_{g}^{+-}(0)\right]\right| P\right\rangle_{\text {nondiag }}=-C_{f} \frac{g^{2}}{2 \pi^{2}} \Lambda^{2} \int_{0}^{1} \frac{d x}{x} \frac{1+x^{2}}{(1-x)^{2}} .
$$

Next let us consider diagonal contributions. From the gauge boson part of the Hamiltonian density, we have,

$$
\left\langle P\left|\theta_{g}^{+-}(0)\right| P\right\rangle_{\text {diag }}-\frac{\left(P^{\perp}\right)^{2}}{\left(P^{+}\right)^{2}}\left\langle P\left|\theta_{g}^{++}(0)\right| P\right\rangle_{\text {diag }}=C_{f} \frac{g^{2}}{4 \pi^{2}} \Lambda^{2} \int_{0}^{1} \frac{d x}{1-x} \frac{1+x^{2}}{1-x} .
$$

Adding the diagonal contributions from the fermion and gauge boson parts, i.e., Eqs. (34) and (39) we arrive at

$$
\left\langle P\left|\theta^{+-}(0)\right| P\right\rangle_{\text {diag }}-\frac{\left(P^{\perp}\right)^{2}}{\left(P^{+}\right)^{2}}\left\langle P\left|\theta^{++}(0)\right| P\right\rangle_{\text {diag }}=C_{f} \frac{g^{2}}{4 \pi^{2}} \Lambda^{2} \int_{0}^{1} \frac{d x}{x} \frac{1+x^{2}}{(1-x)^{2}}
$$

Thus the total contribution (quark and gluons) to the expectation value of the Hamiltonian density is given by

$$
\left\langle P\left|\theta^{+-}(0)\right| P\right\rangle-\frac{\left(P^{\perp}\right)^{2}}{\left(P^{+}\right)^{2}}\left\langle P\left|\theta^{++}(0)\right| P\right\rangle=-C_{f} \frac{g^{2}}{4 \pi^{2}} \Lambda^{2} \int_{0}^{1} \frac{d x}{x} \frac{1+x^{2}}{(1-x)^{2}} .
$$

This result is directly related to the mass shift of the fermion to order $g^{2}$ in light-front perturbation theory. We have in the limit of zero bare mass, (see Eq. (4.10) in Ref. [13]),

$$
\delta p_{1}^{-}=-\frac{1}{2 P^{+}} C_{f} \frac{g^{2}}{4 \pi^{2}} \Lambda^{2} \int_{0}^{1} \frac{d x}{x} \frac{1+x^{2}}{(1-x)^{2}} .
$$


Note that in the massless limit we encountered only quadratic divergences in the twist four part of the longitudinal structure function. It is now obvious that for a massive quark, we will also encounter logarithmic divergences.

To test the sum rule given in Eq. (23) explicitly in a non-perturbative context we turn to two-dimensional QCD. In $1+1$ dimensions, in $A^{+}=0$ gauge, we have,

$$
\int_{0}^{1} \frac{d x}{x} F_{L(q)}^{\tau=4}(x)=\frac{2}{Q^{2}}\left\langle P\left|\left[\theta_{q}^{+-}(0)+\theta_{g}^{+-}(0)\right]\right| P\right\rangle,
$$

with $\theta_{q}^{+-}=2 m^{2} \psi^{+\dagger} \frac{1}{i \partial^{+}} \psi^{+}$and $\theta_{g}^{+-}=-4 g^{2} \psi^{+\dagger} T^{a} \psi^{+} \frac{1}{\left(\partial^{+}\right)^{2}} \psi^{+\dagger} T^{a} \psi^{+}$. In the standard one pair $(q \bar{q})$ approximation to the ground state, with the help of the bound state equation ('t Hooft equation) obeyed by the ground state wave function $\psi(x)$ for the meson

$$
M^{2} \psi(x)=\frac{m^{2}}{x(1-x)} \psi(x)-C_{f} \frac{g^{2}}{\pi} \int d y \frac{\psi(y)-\psi(x)}{(x-y)^{2}}
$$

together with the normalization condition $\int_{0}^{1} d x \psi^{2}(x)=1$, it is easily verified that the twist four longitudinal structure function of the meson obeys the sum rule

$$
\int_{0}^{1} \frac{d x}{x} F_{L}^{\tau=4}=\frac{2}{Q^{2}}\left\langle P\left|\theta^{+-}(0)\right| P\right\rangle=4 \frac{M^{2}}{Q^{2}} .
$$

To summarize, we have investigated the twist four contributions to the longitudinal structure function in deep inelastic scattering in an approach based on Fock space expansion methods in light-front field theory. Our motivation has been to gain physical intuition on the twist four part of the longitudinal structure function and to understand the occurrence of quadratic divergences in $F_{L(q)}^{\tau=4}$ and the associated renormalization issues. We have found that the integral of $\frac{F_{L(q)}^{\tau=4}(x)}{x}$ is related to the expectation value of the fermionic part of the light-front Hamiltonian density. The relation, which is tested to order $g^{2}$ in perturbative QCD in the bare theory, explicitly relates the quadratic divergences generated in $F_{L(q)}^{\tau=4}$ to the fermion mass shift arising from the fermionic part of the light-front Hamiltonian density. By investigating the operator mixing problem, we have derived a new sum rule which relates the integral of $\frac{F_{L}^{\tau=4}(x)}{x}$ to the invariant mass of the hadron. We have explicitly checked the validity of this sum rule in two dimensional QCD.

The phenomenological consequences of the new sum rule are worth studying. Clearly, experiments to measure the twist four longitudinal structure function will reveal the fraction of the hadron mass carried by the charged parton components. These experiments will complement the longitudinal momentum and helicity distribution information obtained at the twist two level. It is of interest to investigate the feasibility of the direct measurement of the twist four gluon structure function in high energy experiments. Recent work of Qiu, Sterman and collaborators have shown that semi-inclusive single jet production in deep inelastic scattering [14] and direct photon production in hadron nucleus scattering [15] provide direct measurement of twist four gluon matrix elements.

On the theoretical side we note that some significant progress has been made recently in the bound state problem in light-front QCD [16]. In the near future, we plan to undertake a non-perturbative calculation (utilizing Fock space expansion and Hamiltonian renormalization techniques) of the longitudinal structure function for a meson-like bound state. Such a 
calculation will undoubtedly help to check the validity of current phenomenological models [2] based on simple assumptions [17] employed in analyzing the data. Whether the relation between the integral of $\frac{F_{L(q)}^{\tau=4}(x)}{x}$ and the hadron expectation value of the fermionic part of the light-front QCD Hamiltonian density can provide insight in to the small $x$ behavior of $F_{L(q)}^{\tau=4}$ is also worth investigating.

One of the authors (AH) would like to thank Wei Min Zhang for useful discussions. This work is supported in part by the U.S. Department of Energy under Grant No. DEFG02 87ER40371, Division of High Energy and Nuclear Physics. 


\section{REFERENCES}

[1] S.D. Dasu et al., Phys. Rev. Lett. 61 (1988) 1061; L.W. Whitlow, S. Rock, A. Bodek, S. Dasu, and E.M. Riordan, Phys. Lett. B 250 (1990) 193.

[2] J.L. Miramontes, M.A. Miramontes, and J. Sanchez Guillen, Phys. Rev. D 40 (1989) 2184.

[3] For an early discussion, see, H.D. Politzer, Nucl. Phys. B 172 (1980) 349.

[4] X. Ji, Nucl. Phys. B 448 (1995) 30.

[5] A. Harindranath and Wei Min Zhang, Phys. Lett. B 390 (1997) 359.

[6] Wei Min Zhang and A. Harindranath, preprint (1996) hep-ph/9606347.

[7] A. Harindranath and Wei Min Zhang, hep-ph/9706419, to appear in Physics Letters B.

[8] For an overview of this approach, see A. Harindranath and Rajen Kundu, preprint (1996) hep-ph/9606433; A. Harindranath, Rajen Kundu, and Wei Min Zhang, in preparation.

[9] R. Jackiw, in Springer Tracts in Modern Physics, Vol. 62, edited by G. Hohler (SpringerVerlag, New York (1972)).

[10] R.K. Ellis, W. Furmanski, and R. Petronzio, Nucl. Phys. B 212 (1983) 29.

[11] J. Qiu, Phys. Rev. D 42 (1990) 30.

[12] In the pre-QCD era this result, for the case of vanishing target transverse momentum, was obtained by D.J. Broadhurst, J.F. Gunion, and R.L. Jaffe, Phys. Rev. D 8 (1973) 566.

[13] Wei Min Zhang and A. Harindranath, Phys. Rev. D 48 (1993) 4881.

[14] M. Luo, J. Qiu, and G. Sterman, Phys. Rev. D 50, (1994) 1951.

[15] X. Guo, and J. Qiu, Phys. Rev. D 53 (1996) 6144.

[16] K.G. Wilson, T.S. Walhout, A. Harindranath, W.-M. Zhang, R.J. Perry, and St. D. Głazek, Phys. Rev. D 49 (1994) 6720; Wei-Min Zhang, Phys. Rev. D 56 (1997) 1528; M. M. Brisudova, R.J. Perry, and K.G. Wilson, Phys. Rev. Lett. 78 (1997) 1227. Also see, S.J. Brodsky, H.-C. Pauli, and S.S. Pinsky, Quantum Chromodynamics and other field theories on the light cone, SLAC-PUB-7484, hep-ph/9705477 and references therein.

[17] R. K. Ellis, W. Furmanski, and R. Petronzio, Nucl. Phys. B 207 (1982) 1. 